California, Berkeley, who carried out the recent VLA observations. "There's a lot we can learn from this."

The magnetar's accidental discovery is a byproduct of astronomers' excitement about the arrival of the gas cloud, dubbed G2. The cloud, which is about three times the mass of Earth, was first spotted near Sgr A* in 2012 (and was later found in 2002 data). Its arrival would deliver insight into how objects accrete into the swirling disk of material around a black hole, as well as offering the first chance for astronomers to measure the time that it takes for objects to be captured and swallowed up.

Every flicker of emissions from Sgr A* sparks a flurry of speculation, intensifying the usual cycle of observation and coordinated follow-up that characterizes high-energy astronomy. Many telescope directors are scheduling additional monitoring of the Galactic Centre. The VLA, for example, is already scanning radio frequencies around $\mathrm{Sgr} \mathrm{A}^{*}$ every two months, and will do so every month once G2 arrives.

"I don't think there was ever such a large camp of telescopes looking at the Galactic Centre," says Stefan Gillessen, an astronomer at the Max Planck Institute for Extraterrestrial Physics in Garching, Germany, who last year reported that G2 was on course for Sgr A* (S. Gillessen et al. Nature 481, 51-54; 2012).

The observing frenzy is likely to jeopardize regularly scheduled observations, and some astronomers worry that the pay-off may be disappointing. Andrea Ghez, an astronomer studying G2 at the University of California, Los Angeles, says that her infrared observations at the Keck telescopes on Mauna Kea in Hawaii suggest that G2 may not be a gas cloud, but rather a star surrounded by gas. If she is right, Sgr $\mathrm{A}^{\star}$ may swallow some of the gas, but the star itself would have enough momentum to escape the black hole's grasp. The result would be a Galactic fizzle rather than fireworks. "I'm just worried that this is overblown," she says.

Yet the focus on the Galactic Centre has paid off unexpectedly. The reported X-ray flare prompted Fiona Harrison, an astronomer who leads NASA's Nuclear Spectroscopic Telescope Array (NuSTAR) satellite, to train the X-ray space telescope on Sgr $A^{*}$. Like Frail, Harrison doubted that the flare had anything to do with G2, but she thought that NuSTAR's ability to time events precisely might clarify the situation. Sure enough, on 26 April, NuSTAR detected an X-ray signal blinking every 3.76 seconds - the signal of a pulsar spinning at that rate. Then, on 29 April, the Chandra $\mathrm{X}$-ray Observatory localized the magnetar to at least 0.12 parsecs ( 0.38 light years) from the black hole - close, but much farther away than G2.

On 4 May, observations by NuSTAR and Swift, the NASA space telescope that spotted the original flare, identified the object conclusively as a magnetar by showing a gradual slowing in its spin rate. This slowing is consistent with the presence of the high magnetic field that enables the star to radiate energy faster than a normal pulsar.

Fourteen magnetars, including this one, have been found in the general region of the Galactic Centre. This high number supports the idea that magnetars tend to form from the death throes of the bright, heavy stars that are common there, says Chryssa Kouveliotou, a magnetar expert at NASA's Marshall Space Flight Center in Huntsville, Alabama.

The finding also hands astronomers a tool for studying conditions

\section{"Idon't think}

there was

ever such a

large camp

of telescopes

looking at the

Galactic Centre."

time. So if the mag-
netar is following an elliptical orbit around the black hole, its clock-like spin rate should speed up and slow down as its distance from the black hole varies - an effect that, with luck, could be disentangled from the gradual slow-down caused by the magnetar's magnetic field.

Frail suggests that the magnetar's discovery shows that all the excitement over G2 is justified. Astronomers watching G2 say that the cloud itself could deliver plenty more excitement when it finally reaches the Galactic Centre. They are undaunted by the fact that one of the first apparent signs of its arrival turned out to be something completely different. "I wish all of our failed experiments were that good," says Frail.

\title{
Chinese project probes the genetics of genius
}

\section{Bid to unravel the secrets of brainpower faces scepticism.}

\section{BY ED YONG}

$\mathrm{T}$ The US adolescents who signed up for the Study of Mathematically Precocious Youth (SMPY) in the 1970s were the smartest of the smart, with mathematical and verbal-reasoning skills within the top $1 \%$ of the population. Now, researchers at BGI (formerly the Beijing Genomics Institute) in Shenzhen, China, the largest gene-sequencing facility in the world, are searching for the quirks of DNA that may contribute to such gifts. Plunging into an area that is littered with failures and riven with controversy, the researchers are scouring the genomes of 1,600 of these high-fliers in an ambitious project to find the first common genetic variants associated with human intelligence.

The project, which was launched in August 2012 and is slated to begin data analysis in the next few months, has spawned wild accusations of eugenics plots, as well as more measured objections by social scientists who view such research as a distraction from pressing societal issues. Some geneticists, however, take issue with the study for a different reason. They say that it is highly unlikely to find anything of interest - because the sample size is too small and intelligence is too complex.

Earlier large studies with the same goal have failed. But scientists from BGI's Cognitive Genomics group hope that their super-smart sample will give them an edge, because it should be enriched with bits of DNA that confer effects on intelligence. "An exceptional person gets you an order of magnitude more statistical power than if you took random people from the population - I'd say we have a fighting chance," says Stephen Hsu, a theoretical physicist from Michigan State University in East Lansing, who acts as a scientific adviser to BGI and is one of the project's leaders.

"If they think they're likely to get much useful data out of this study, they're almost certainly wrong," says Daniel MacArthur, a geneticist at Massachusetts General Hospital in Boston. He is not against intelligence 


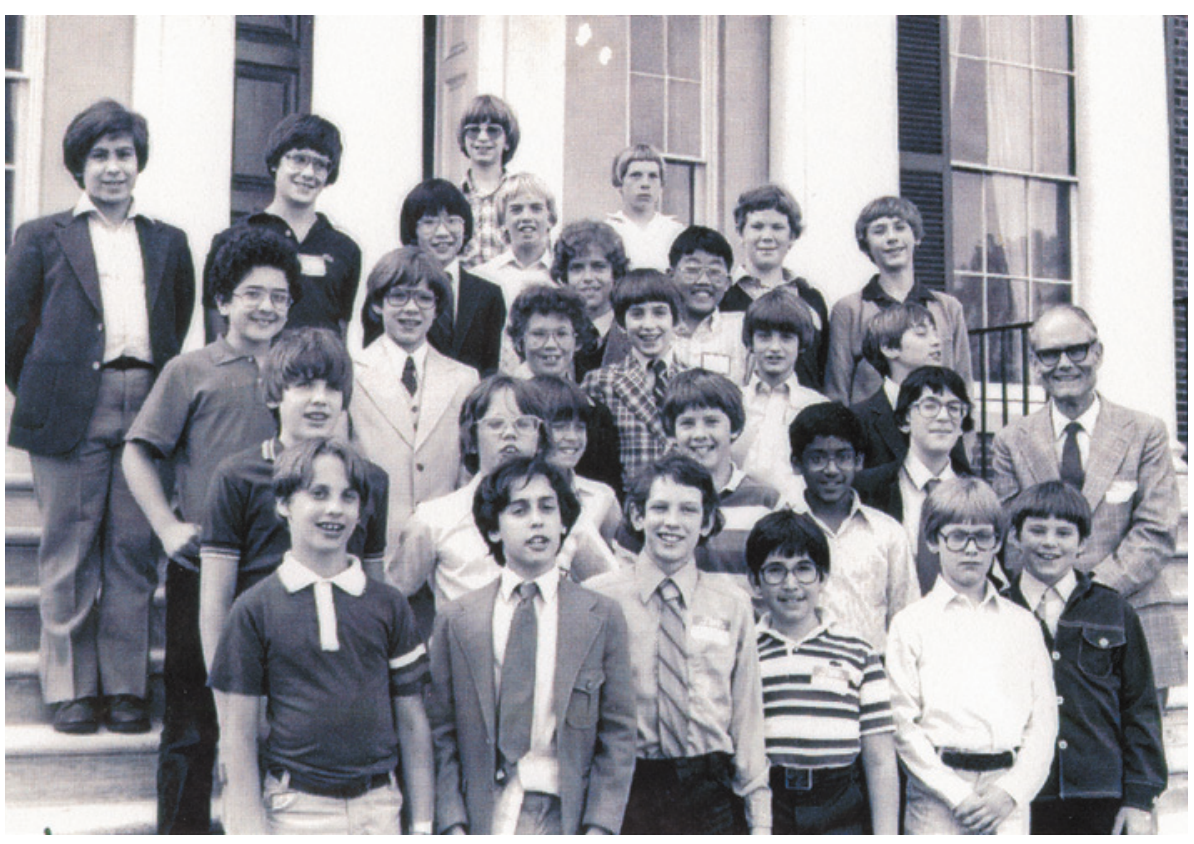

Candidates for the Study of Mathematically Precocious Youth in 1983. Study data are in use at China's BGI.

studies in principle, despite the visceral reactions they provoke in some people. "Studying intelligence is useful for understanding cognitive function, or diseases" that affect it, he says. But he questions whether the study will work.

Intelligence has a substantial but mysterious genetic component ${ }^{1}$. Studies in twins indicate that genetic factors should explain significantly more than half of the variation in adult general intelligence - the abstract quantity measured in IQ tests. This, in turn, correlates well with attributes such as academic achievement and income. Although geneticists have identified hundreds of genes in which a single mutation can lead to developmental difficulties, the common genetic variants that lie behind normal diversity in intelligence remain elusive. It is widely assumed that, as is the case for traits such as height, there are thousands of such variants, the small effects of which combine to influence mental abilities.

So far, the quest to identify intelligencelinked variants has thrown up only null results and false positives ${ }^{2}$, even after large, well-designed studies. In 2010, a team led by Robert Plomin, a behavioural geneticist at King's College London, failed to find a single intelligence-associated variant, even after

examining more than 350,000 variations in single DNA letters, or SNPs, across the genomes of 7,900 children ${ }^{3}$.

After this, Plomin switched his strategy to focus on only the brightest minds. He collected DNA samples from 2,000 of the SMPY's recruits, whose average IQ is above 150 - surpassing the average of Nobel laureates and putting them three standard deviations above the general population's mean score of 100 . "In the earlier study, I bet we didn't have more than two or three people with an IQ that high," says Plomin, who has been studying the heritability of intelligence since the 1970s.

At the same time,

"If they think they're likely to get much useful data out of this study, they're almost certainly wrong."

BGI had hit on a similar strategy and was trying to recruit the brightest teenagers for a new study. Although Hsu is not a geneticist himself, he has a long-standing interest in intelligence. He suggested that BGI should recruit gifted individuals from science and mathematics olympiads - international competitions for school pupils - but when that approach failed, Hsu set up a website to attract volunteers of 'high cognitive ability'.
Then he heard about Plomin's sample. The two struck up a partnership: Plomin supplied DNA samples from 1,600 SMPY recruits, and Hsu added samples from more than 500 people recruited - albeit less selectively - through his website, which asked potential donors to submit evidence of their brain power in the form of impressive olympiad results, high scores on standardized tests or diplomas from leading universities. "We're at the bleeding edge," says Hsu. "No one has a sample like this."

BGI is sequencing the genomes at low coverage - scanning each DNA letter an average of only four or five times - and comparing them with what Hsu says will be in excess of 4,000 controls from the UK10K project, which aims to sequence the genomes of 10,000 UK residents. Because these people were not selected for intelligence, they should display a range of IQs. The researchers at BGI will begin by using commercially available tools to look at common SNPs before moving on to rarer ones, and to variations in the number of copies of different DNA segments.

Plomin is optimistic about the study's chances: "I'm not expecting to find a lot but I'd be happy with one solid finding," he says. But MacArthur notes that searches for genes connected with schizophrenia have had similar sample sizes and compared people at the far end of the risk spectrum (extremely likely to get the disease) with those in the middle (of average risk). Yet the efforts came up emptyhanded. If the genetics of intelligence are similar to those of schizophrenia or height, MacArthur says, the team needs at least 10,000 cases and 10,000 controls.

Sample size could be an even greater handicap if it turns out that intelligence is not actually the result of multiple variants, each with a small effect. Plomin likens a super-smart brain to a finely tuned race car: "You need many positive things going for you and you can't afford many negatives," he says.

But this may not be true, says Kevin Mitchell, a neurogeneticist from Trinity College Dublin. "Intellectual disability can be caused by a mutation in any one of a large number of genes - we don't know if that is true for exceptional genius," he says. If rare mutations produce extremes in intelligence just as they do extremes in height, the BGI study will be unlikely to find them.

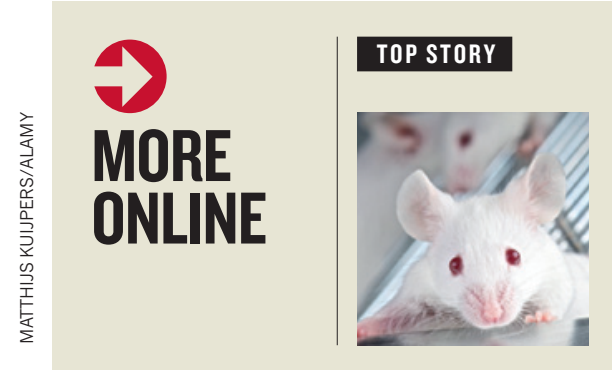

Blood hormone relieves agerelated heart stiffening and thickening in mice gonature. com/qsulug

\section{MORE NEWS}

- Crowdsourcing project set to locate world's power plants go.nature.com/eoasws - Official data contradict Spanish government's claims on science funding go.nature.com/3clk6k

- Common origin for Earth and Moon water go.nature.com/mi2urw

\section{VIDEO OF THE WEEK}

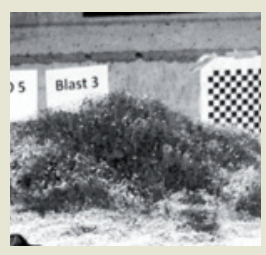

Artificial volcanoes offer insight into explosions go.nature.com/ yvcen? 
Both Plomin and Hsu are passionate enough to take a shot, although their goals differ. Hsu is focused on the genetic basis of extreme intelligence. "My primary interest is why Einstein or Hawking is different from a normal person," he says. Plomin is sequencing high-performers as a way of homing in on genes that affect intelligence in the broader population. If enough of these are discovered, he thinks that it may be possible to predict someone's intelligence from an early age, and to offer help to children who are at risk of learning disabilities.

Publicity around the project has spawned some extreme reactions. An article published in March entitled 'China is Engineering Genius Babies' in the US arts and culture magazine VICE branded the study "a state-endorsed genetic-engineering project" that will allow parents to predict the IQs of embryos and selectively breed eversmarter children. ("That's nuts," says Hsu.) "Intelligence does push a lot of buttons. It's like waving a red flag to a bull," says Plomin. $\mathrm{He}$ argues that there is nothing wrong with using genetic information as the basis of educational interventions. "I'm interested in predicting learning problems early rather than waiting until kids get to school and then fail," he says.

But Paul Martin, a sociologist at the University of Sheffield, UK, is surprised that geneticists are still pursuing this line of research. "I think most people would say that's the wrong paradigm, when most educational research suggests that social factors are incredibly important," he says. "Strategically, this seems like something of a throwback."

More controversy is likely to flare when or if an intelligence factor emerges from the project. BGI is halfway through its sequencing, but it is unclear when findings will be released. "You might not see anything for a year," says Hsu; it depends on how long the analysis takes. In the meantime, as the cost of sequencing continues to fall, larger sample sizes may soon become available for studies of this kind. "In 2009, we thought it'd be impossible to have a schizophrenia study with 20,000 cases," says Peter Visscher, a geneticist from the University of Queensland in Brisbane, Australia. "Now, the international community is up to 40,000 ."

Still, he adds that the idea of predicting intelligence from a DNA sample is fanciful. "Even for human height, where you have samples of hundreds of thousands, the prediction you'd get for a newborn person isn't very accurate," he says. "That will be true for IQ for a long time to come." -

1. Deary, I. J., Johnson, W. \& Houlihan, L. M. Hum. Genet. 126, 215-232 (2009)

2. Chabris, C. F. et al. Psychol. Sci. 23, 1314-1323 (2012).

3. Davis, O. S. et al. Behav. Genet. 40, 759-767 (2010).

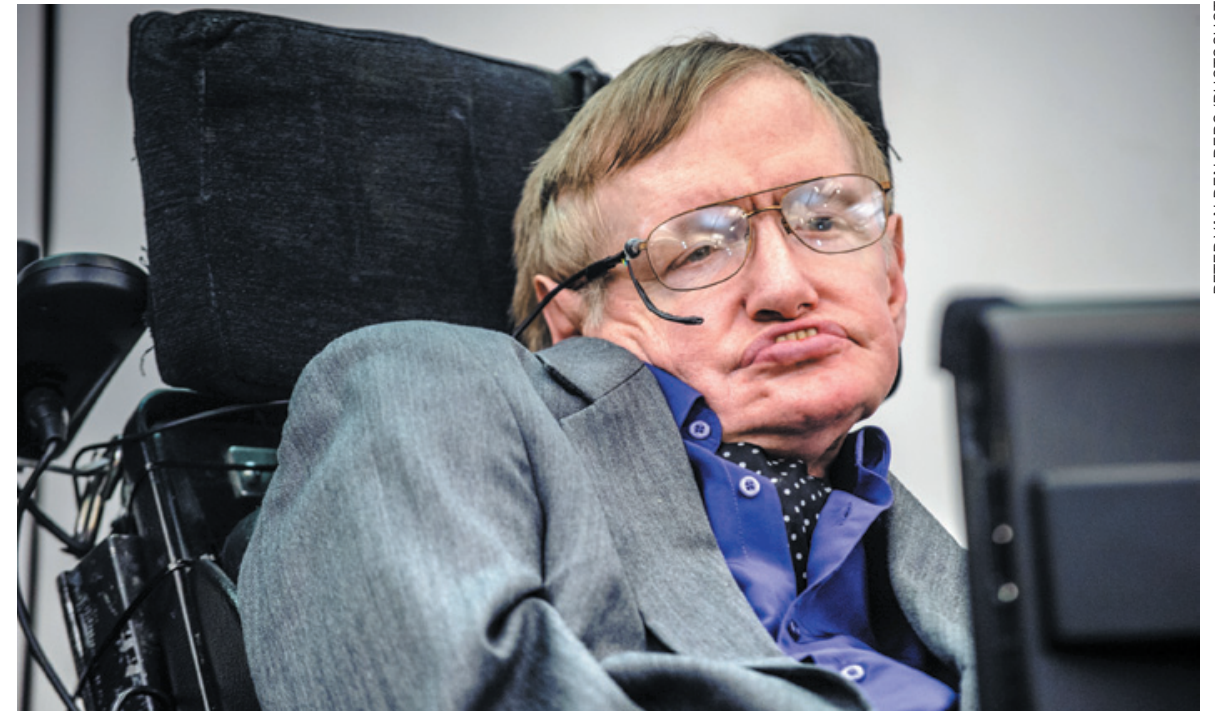

Stephen Hawking joins a list of scientists who have boycotted Israeli institutions and events.

\section{ACADEMIC BOYCOTTS}

\section{Hawking decision fuels Israel debate}

\section{Physicist's stance a 'turning point', claim supporters.}

\section{BY DANIEL CRESSEY}

A decision by one of the world's most famous physicists to withdraw from a conference due to be held in Israel next month has rekindled fierce debate in the United Kingdom over academic boycotts that are intended to protest against Israel's actions in the occupied Palestinian territories.

The decision, by Stephen Hawking of the University of Cambridge, UK, comes hot on the heels of an official ruling against a UK academic who had argued that, in part by repeatedly discussing a boycott of Israel, his trade union had created a hostile and anti-Semitic environment. Observers expect that the ruling will add fuel to this contentious fight.

Hawking, director of research at the Centre for Theoretical Cosmology, was scheduled to attend the Israeli Presidential Conference in Jerusalem, an event run in partnership with the Hebrew University of Jerusalem that will also celebrate the 90th birthday of Israeli President Shimon Peres. The gathering on 18-20 June will feature talks by scientists, other academics, artists and politicians on topics ranging from education and new media to political leadership.

Hawking wrote to the conference organizers on 3 May to say that he would not be attending so as to "respect the boycott" that some researchers have called for over the past decade. His decision was announced last week on the website of the British Committee for the Universities of Palestine, a London-based group calling for academics to cut links to Israeli institutions, and triggered intense media attention.

The University of Cambridge initially said in a statement released last Wednesday that the physicist, who has the debilitating neurological condition motor-neuron disease, was withdrawing for health reasons. Later that day, it said that it had been mistaken and that Hawking's decision was "based on advice from Palestinian academics that he should respect the boycott".

Conference organizer Israel Maimon, a lawyer, thinks that Hawking's decision was wrong, and says that attempts to create an academic boycott of Israel are "outrageous and improper".

Such efforts stretch back a decade in Britain. In 2002, more than 100 academics signed a letter published in The Guardian newspaper calling for a moratorium on European funding of, and contracts with, Israeli institutions, in protest at the "violent repression against the Palestinian people in the occupied territories". Since then, various campaign groups and trade unions of teachers and lecturers have proposed boycott motions at their meetings, some of which have been approved.

Steven Rose, an emeritus professor of 